3. B. R. Gelbaum, Tensor products of Banach algebras, Canad. J. Math. 11 (1959), 297-310.

4. J. Tomiyama, Tensor products of commutative Banach algebras, Tôhoku Math. J. 12 (1960), 147-154.

5. R. Schatten, $A$ theory of cross spaces, Annals of Mathematics Studies No. 26, Princeton Univ. Press, Princeton, N. J., 1950.

6. A. Grothendieck, Produits tensoriels topologiques et espaces nucléaires, Mem. Amer. Math. Soc. No. 16, 1955.

7. J. Gil de Lamadrid, Measures and tensors, (to appear).

8. L. Loomis, Introduction to abstract harmonic analysis, Van Nostrand, New York, 1953.

UNIVERSITY OF MiNNESOTA

\title{
AN INTEGRATION-BY-PARTS FORMULA ${ }^{1}$
}

BY J. S. MAC NERNEY

Communicated by Walter Rudin, August 5, 1963

In 1914, W. H. Young [4] introduced a modification of the Riemann-Stieltjes integral which, for functions $F$ and $G$ defined on the real line with $G$ of bounded variation on each interval and $F$ suitably restricted, yields an additive interval function:

$$
\text { (Y) } \int_{a}^{b} F \cdot d G+(Y) \int_{b}^{c} F \cdot d G=(Y) \int_{a}^{c} F \cdot d G .
$$

In 1959, T. H. Hildebrandt [1] published a study of a certain linear initial-value problem involving these Young integrals, which extended some of the earlier results of $\mathrm{H}$. S. Wall and of the present author (see [2] for discussion and references). In 1962, there was discovered a connection between the Young integral and the interior integral as introduced by S. Pollard in 1920 [3], viz., the systems

$$
U(x)=U(c)+(Y) \int_{0}^{x} U \cdot d H \text { and } V(x)=V(c)+(I) \int_{x}^{c} d H \cdot V,
$$

with $H$ a function from the real line to a complete normed ring, are naturally adjoint to one another [2, p. 326]. Both integrals are to be interpreted as limits in the sense of successive refinements of subdivisions of the interval of integration.

Suppose each of $F$ and $G$ is a function from the real line to the complete normed ring $N$. If each of $F$ and $G$ is of bounded variation

\footnotetext{
1 Presented to the Society, July 18, 1963.
} 
on the interval $[a, b]$ then each of $(Y) \int_{a}^{b} F \cdot d G$ and $(I) \int_{a}^{b} d F \cdot G$ is known to exist. Hence, the latter integral exists under the condition that $F$ is of bounded variation on $[a, b]$ and $G$ is quasicontinuous, i.e., each of the limits $G(x-)$ and $G(x+)$ exists for each number $x$. Here is a new connection between these integrals, which also provides a new existence theorem for the former one.

THEOREM A. If each of $F$ and $G$ is a function from the real line to the complete normed ring $N, F$ is of bounded variation on the interval $[a, b]$, and $G$ is quasicontinuous, then

$$
(Y) \int_{a}^{b} F \cdot d G+(I) \int_{a}^{b} d F \cdot G=F(b) G(b)-F(a) G(a) .
$$

INDICATION OF PROOF. If $a \leqq x<y<z \leqq b$ then

$$
\begin{aligned}
W= & F(x)[G(x+)-G(x)]+F(y)[G(z-)-G(x+)]+F(z)[G(z)-G(z-)] \\
& +[F(z)-F(x)] G(y)-[F(z) G(z)-F(x) G(x)] \\
= & {[F(x)-F(z)][G(x+)-G(y)]+[F(z)-F(y)][G(x+)-G(z-)], }
\end{aligned}
$$

so that one has the estimate

$$
\left.|W| \leqq 2\left(\int_{x}^{z}|d F|\right) \text { (L.U.B. }|G(v)-G(u)| \text { for } x<u<v<z\right) \text {. }
$$

Addendum. As has been observed by Randolph Constantine (an oral communication in seminar), the hypotheses on $F$ and $G$ in Theorem A can be interchanged. To see this, one first notes the identity

$$
\begin{aligned}
& {[F(z)-F(x)] G(y)} \\
& \quad=F(z) G(z)-F(x) G(x)-F(x)[G(y)-G(x)]-F(z)[G(z)-G(y)] ;
\end{aligned}
$$

next, if $H$ is a simple step-function and $\left\{t_{p}\right\}_{0}^{2 n}$ is an increasing numerical sequence with $t_{0}=a$ and $t_{2 n}=b$,

$$
\begin{aligned}
& \left|\sum_{1}^{n}\left[F\left(t_{2 p}\right)-F\left(t_{2 p-2}\right)\right] G\left(t_{2 p-1}\right)-\sum_{1}^{n}\left[H\left(t_{2 p}\right)-H\left(t_{2 p-2}\right)\right] G\left(t_{2 p-1}\right)\right| \\
& \leqq|F-H|_{[a, b]}\left(|G(a)|+|G(b)|+\int_{a}^{b}|d G|\right),
\end{aligned}
$$

where $|F-H|_{[a, b]}=$ L.U.B. $|F(u)-H(u)|$ for $u$ in $[a, b]$, and also

$$
\left|(Y) \int_{a}^{b} F \cdot d G-(Y) \int_{a}^{b} H \cdot d G\right| \leqq|F-H|_{[a, b]}\left(\int_{a}^{b}|d G|\right) .
$$


Thus, an argument is easily made to establish the following somewhat stronger theorem.

THEOREM B. If each of $F$ and $G$ is a quasicontinuous function from the real line to the complete normed ring $N$, and one of $F$ and $G$ is of bounded variation on the interval $[a, b]$, then

$$
\text { (Y) } \int_{a}^{b} F \cdot d G+(I) \int_{a}^{b} d F \cdot G=F(b) G(b)-F(a) G(a) .
$$

REMARK. The reader is invited to contrast this formula with the corresponding result involving Young integrals alone (or interior integrals alone), as obtained by Hildebrandt [1, p. 355] for the case that both $F$ and $G$ are of bounded variation. For this case, there is a more general result available, as indicated in the following theorem.

Theorem C. If Axioms I and II [2, p. 321] hold, each of $F$ and $G$ is a function from the interval $[a, b]$ to $N$, and $d G(x, z)=K_{1}[1](x, z)$ and $d F(x, z)=K_{2}[1](x, z)$ for $a \leqq x<z \leqq b$, then

$$
\begin{aligned}
& K_{1}[F](a, b)+K_{2}[G](a, b) \\
& =F(b) G(b)+\sum_{a<z \leqq b}\left\{d F \cdot K_{1}\left[1_{z}\right]+K_{2}\left[1_{z}\right] \cdot d G-d F \cdot d G\right\}(z-, z) \\
& \quad-F(a) G(a)-\sum_{a \leqq x<b}\left\{d F \cdot K_{1}\left[1_{x}\right]+K_{2}\left[1_{x}\right] \cdot d G-d F \cdot d G\right\}(x, x+) .
\end{aligned}
$$

REMARK. After obtaining the preceding results, the author learns (July 27,1963 ) that Theorem B has been discovered by T. H. Hildebrandt (on May 28, 1963) for numerical valued functions $F$ and $G$ : that priority of discovery is hereby cordially acknowledged to Professor Hildebrandt.

\section{BIBLIOGRAPHY}

1. T. H. Hildebrandt, On systems of linear differentio-Stieltjes-integral equations, Illinois J. Math. 3 (1959), 352-373.

2. J. S. Mac Nerney, A linear initial-value problem, Bull. Amer. Math. Soc. 69 (1963), 314-329.

3. S. Pollard, The Stieltjes integral and its generalizations, Quart. J. Pure and Appl. Math. 49 (1920), 73-138.

4. W. H. Young, On integration with respect to a function of bounded variation, Proc. London Math. Soc. (2) 13 (1914), 109-150.

The University of North Carolina at Chapel Hill 